B.E. RHOADES

KODAI MATH. J.

4 (1981). 293-297

\title{
A FIXED POINT THEOREM FOR ASYMPTOTICALLY NONEXPANSIVE MAPPINGS
}

\author{
By B. E. RHOADES
}

Let $H$ be a real Hilbert space, $C$ a closed convex subset of $H, T$ a selfmapping of $C$. Let $A_{n} x$ denote the $n$th term of the Cesàro transform of the sequence of iterates $\left\{T^{k} x\right\}$. Baillon [1] proved that, if $T$ is a nonexpansive selfmapping of $C$ which has a fixed point, then $\left\{A_{n} x\right\}$ converges weakly to a fixed point of $T$. This result has been extended to strongly regular matrices by Brézis and Browder [2], Bruck [3], and Reich [9]. In a recent paper [6] the Baillon result was extended to asymptotically nonexpansive mappings. In this paper the result of $[6]$ is extended to a wide class of matrix methods.

A mapping $T$ is said to be asymptotically nonexpansive over $C$ if, for each $x, y \in C$,

$$
\left\|T^{2} x-T^{i} y\right\| \leqq\left(1+\alpha_{\imath}\right)\|x-y\|, \quad \imath=1,2, \cdots,
$$

where $\lim _{i} \alpha_{\imath}=0$.

An infinite matrix $A=\left(a_{n_{k}}\right)$ is called regular if it is limit-preserving over $c$, the space of convergent sequences. Necessary and sufficient conditions for regularity are: (i) $\|A\|=\sup _{n} \sum_{k=0}^{\infty}\left|a_{n k}\right|<\infty$; (ii) $\lim _{n} a_{n k}=0$ for $k=0,1,2, \cdots$, and (iii) $\lim _{n} t_{n}=1$, where $t_{n}=\sum_{k=0}^{\infty} a_{n k}$. Let $X$ be a locally convex space. A sequence $\left\{x_{n}\right\} \subset X$ is said to be almost convergent, written $a c$, if there exists a point $s \in X$ such that $\lim _{n} \sum_{k=0}^{n-1} x_{k+\imath} / n=s$, uniformly in 2 . A matrix $A$ will be called strongly regular if, in addition to satisfying conditions (i) and (iii) for regularity, it also satisfies (ii') $\lim _{n} \sum_{k}\left|a_{n k}-a_{n, k+1}\right|=0$. A is called triangular if all its entries above the main diagonal are zero.

THEOREM. Let $C$ be a closed convex subset of a real Hilbert space $H, T$ an asymptotically nonexpansive selfmap of $C$ such that $\left\{T^{n} z\right\}$ is bounded for each $z \in C$. Let $A$ be a strongly regular matrix. Define $A_{n} x=\sum_{k=0}^{\infty} a_{n k} T^{k} x$. Then, for each $x \in C,\left\{A_{n} x\right\}$ converges weakly to a fixed point $p$, which is the asymptotic center of $\left\{T^{n} x\right\}$.

The proofs of Lemmas 2 and 3 of [6] are independent of the matrix $A$ involved. So, to prove the Theorem, it is sufficient to show that Lemma 1 of [6] is true for each strongly regular matrix $A$; i. e., there exists a positive integer

Received February 28, 1980 
$K_{0}$ such that, for each $m \geqq K_{0}$, there exists a positive integer $N_{m}$ satisfying

$$
\left\|A_{n} x-T^{m} A_{n} x\right\|<\varepsilon \quad \text { for all } n \geqq N_{m} .
$$

A regular matrix $A$ will be called a hump matrix if each row contains a hump, and the heights of the humps tend to zero; i. e., for each $n$ there exists an integer $p(n)$ such that $a_{n k} \leqq a_{n, k+1}$ for $0 \leqq k<p$ and $a_{n k} \geqq a_{n, k+1}$ for $k \geqq p$, and $\lim _{n} \sup _{k}\left|a_{n k}\right|=0$.

The proof of (2) will make use of the following Lemma, which is an improvement of Lemma 1.1 of [3].

LEMma. Let $X$ be a sequentially complete space. Then the following are equivalent :

(i) A sequence $x \in X$ is $a c$,

(ii) $\lim _{n} \sum_{k} a_{n k} x_{k}$ exists for every strongly regular matrix $A$,

(iii) $\lim _{n} \sum_{k} a_{n k} x_{k}$ exists for every regular hump matrix $A$,

(iv) $\lim _{n} \sum_{k} a_{n k} x_{k}$ exists for every regular tranglar hump matrix $A$ with nonnegatıve entries and row sums one.

The equivalence of (i) and (ii) comes from [3]. The implications (ii) $\Rightarrow$ (iii) $\Rightarrow$ (iv) are clear, since a regular hump matrix is also strongly regular. In [10] it was shown that (iii) $\Rightarrow$ (i), but the proof there uses matrices satisfying (iv). Therefore (iv) $\Rightarrow$ (i).

From the Lemma, it is sufficient to establish (2) for triangular regular hump matrices with nonnegative entries and row sums one.

For any $u \in H$,

$$
\begin{aligned}
\left\|A_{n} x-u\right\|^{2} & =\left\|\sum_{k=0}^{n} a_{n k} T^{k} x-u\right\|^{2}=\left\|\sum_{k=0}^{n} a_{n k}\left(T^{k} x-u\right)\right\|^{2} \\
& =\sum_{k=0}^{n} \sum_{\jmath=0}^{n} a_{n k} a_{n \jmath}\left(T^{k} x-u, T^{\jmath} x-u\right) .
\end{aligned}
$$

Since $H$ is a real Hilbert space, $2\left(T^{k} x-u, T^{\jmath} x-u\right)=\left\|T^{k} x-u\right\|^{2}+\left\|T^{\jmath} x-u\right\|^{2}$ $-\left\|T^{k} x-T^{\jmath} x\right\|^{2}$, so that

$$
2\left\|A_{n} x-u\right\|^{2}=2 \sum_{k=0}^{n} a_{n k}\left\|T^{k} x-u\right\|^{2}-\sum_{k=0}^{n} \sum_{\jmath=0}^{n} a_{n k} a_{n \jmath}\left\|T^{k} x-T^{\jmath} x\right\|^{2} .
$$

Substituting $u=A_{n} x$ in (3) yields

$$
\sum_{k=0}^{n} \sum_{j=0}^{n} a_{n k} a_{n j}\left\|T^{k} x-T^{\jmath} x\right\|^{2}=2 \sum_{k=0}^{n} a_{n k}\left\|T^{k} x-A_{n} x\right\|^{2} .
$$

Put (4) in (3) and set $u=T^{k} A_{n} x$ to get, for $k \leqq n$, 


$$
\begin{aligned}
\left\|A_{n} x-T^{k} A_{n} x\right\|^{2}= & \sum_{j=0}^{k-1} a_{n j}\left\|T^{\jmath} x-T^{k} A_{n} x\right\|^{2} \\
& +\sum_{j=k}^{n} a_{n j}\left\|T^{\jmath} x-T^{k} A_{n} x\right\|^{2}-\sum_{j=0}^{n} a_{n j}\left\|T^{\jmath} x-A_{n} x\right\|^{2} .
\end{aligned}
$$

Using (1), and the fact that $a_{n k} \geqq 0$, it then follows that

$$
\begin{aligned}
\left\|A_{n} x-T^{k} A_{n} x\right\|^{2} \leqq & \sum_{j=0}^{k-1} a_{n j}\left\|T^{\jmath} x-T^{k} A_{n} x\right\|^{2} \\
& +\left(2 \alpha_{k}+\alpha_{k}{ }^{2}\right) \sum_{j=0}^{n-k} a_{n, j+k}\left\|T^{\jmath} x-A_{n} x\right\|^{2} \\
& +\sum_{j=0}^{n-k}\left(a_{n, j+k}-a_{n j}\right)\left\|T^{\jmath} x-A_{n} x\right\|^{2} \\
= & I_{1}+I_{2}+I_{3}, \quad \text { say. }
\end{aligned}
$$

By hypothesis $\left\{T^{\jmath} x\right\}$ is bounded for each $x \in C$. Let $M=\sup \left\{\left\|T^{\jmath} x\right\|: \jmath=0,1\right.$, $2, \cdots\}$. Since $A$ is nonnegative with row sums one, $\left\|A_{n} x\right\| \leqq M$.

Since $A$ is a hump matrix, $a_{n, j+1}-a_{n_{j}} \leqq 0$ for $j \geqq p$.

An estimate will first be found for $I_{3}$.

Case I. Suppose $p \leqq k<n-k$. Then

$$
\begin{aligned}
I_{3} & \leqq \sum_{j=0}^{p}\left(a_{n, j+k}-a_{n j}\right)\left\|T^{\jmath} x-A_{n} x\right\|^{2} \\
& \left.\leqq \sum_{j=0}^{p}\left(a_{n p}-a_{n 0}\right) \| T^{\jmath} x-A_{n} x\right) \|^{2} \leqq 4 M^{2} k a_{n p} .
\end{aligned}
$$

Case II. $k<p \leqq n-k$. Then

$$
\begin{aligned}
I_{3} & \leqq \sum_{\jmath=0}^{p-k}\left(a_{n, \jmath+k}-a_{n j}\right)\left\|T^{\jmath} x-A_{n} x\right\|^{2}+\sum_{\jmath=p-k+1}^{p}\left(a_{n, j+k}-a_{n p}+a_{n p}-a_{n j}\right)\left\|T^{\jmath} x-A_{n} x\right\|^{2} \\
& \leqq 4 M^{2}\left[\sum_{\jmath=0}^{p-k}\left(a_{n, j+k}-a_{n j}\right)+0+k a_{n p}-\sum_{\jmath=p-k+1}^{p} a_{n \jmath}\right] \\
& =4 M^{2}\left[\sum_{\jmath=k}^{p} a_{n \jmath}-\sum_{\jmath=0}^{p} a_{n \jmath}+k a_{n p}\right]=4 M^{2}\left[-\sum_{\jmath=0}^{k-1} a_{n \jmath}+k a_{n p}\right] \leqq 4 M^{2} k a_{n p} .
\end{aligned}
$$

Case III. $n-k<p \leqq n$. Then

$$
I_{3}=\sum_{j=0}^{p-k}\left(a_{n, j+k}-a_{n j}\right)\left\|T^{\jmath} x-A_{n} x\right\|^{2}+\sum_{\jmath=p-k+1}^{n-k}\left(a_{n, j+k}-a_{n p}+a_{n p}-a_{n \jmath}\right)\left\|T^{\jmath} x-A_{n} x\right\|^{2} .
$$

Proceeding as in Case II again yields $I_{3} \leqq 4 M^{2} k a_{n p}$. 
Case IV. $p \leqq n-k<k$. Then

$$
I_{3} \leqq \sum_{j=0}^{p}\left(a_{n, j+k}-a_{n j}\right)\left\|T^{\jmath} x-A_{n} x\right\|^{2},
$$

and, following the same argument as Case II, one obtains $I_{3} \leqq 4 M^{2} k a_{n p}$.

Case V. $n-k<p \leqq k$. Then

$$
\begin{aligned}
I_{3} & =\sum_{\jmath=0}^{n-k}\left(a_{n, \jmath+k}-a_{n p}+a_{n p}-a_{n \jmath}\right)\left\|T^{\jmath} x-A_{n} x\right\|^{2} \\
& \leqq 4 M^{2}\left[0+(n-k+1) a_{n p}-\sum_{\jmath=0}^{n-k} a_{n \jmath}\right] \leqq 4 M^{2} k a_{n p} .
\end{aligned}
$$

Case VI. $n-k<k<p \leqq n$. Then $I_{3}$ is the same as in Case V. Thus, in all cases,

$$
I_{3} \leqq 4 M^{2}\left[\sum_{j=0}^{k-1} a_{n j}+k a_{n p}\right] .
$$

Now fix $\varepsilon>0$, and choose $K_{0}$ so that, for all $k \geqq K_{0}, 2 \alpha_{k}+\alpha_{k}{ }^{2}<\varepsilon^{2} / 12 M^{2}$. Then, for all $k \geqq K_{0}$,

$$
I_{2} \leqq 4\left(2 \alpha_{k}+\alpha_{k}^{2}\right) M^{2} \sum_{j=0}^{n} a_{n, j+k} \leqq \varepsilon^{2} / 3 .
$$

Since $A$ is a regular hump matrix, $\lim _{n} a_{n p}=0$ and $A$ has a zero column limits. For each $m \geqq K_{0}$ choose $N_{m}$ so that, for $n \geqq N_{m}, I_{1}<\varepsilon^{2} / 3$ and $I_{3}<\varepsilon^{2} / 3$, and the theorem is proved.

Remarks 1. A closed convex subset $C$ of a real Hilbert space $H$ is said to have the fixed point property for a family $F$ of selfmaps of $C$ if, for every $T \in F$, $T$ has a fixed point. Ray [8] has shown that $C$ has the fixed point property for nonexpansive maps if and only if $C$ is bounded. This result is extendable to asymptotically nonexpansive mappings. The necessity follows by using the same example as in [8], since every nonexpansive mapping is asymptotically nonexpansive. For the sufficiency, assume that $C$ is bounded. Then, from [4, Theorem 1], $T$ has a fixed point in $C$. Consequently, the hypothesis, in the Theorem of this paper, that $\left\{T^{n} z\right\}$ be bounded for $z \in C$ is a natural and necessary one.

2. There are many strongly regular nonnegative matrices for which the Theorem applies. For example the Cesàro matrices of order $\alpha>0$; the Euler matrices; all Nörlund matrices with $p_{n+1} \geqq p_{n}$; and all weighted mean matrices with $p_{n+1} \leqq p_{n}$; all Nörlund matrices with $p_{n+1} \leqq p_{n}$ and $P_{n} \rightarrow \infty$; and by all weighted mean methods with $p_{n+1} \geqq p_{n}$ and $\lim _{n} p_{n} / P_{n}=0$. For definitions and basic properties of these methods the reader may consult [5]. The Chebyshev method (see [7] also satisfies the Theorem.

3. Since $T$ is not assumed to be linear, one obtains a collection of nonlinear 
Ergodic theorems by simply adding the restriction that $t_{n}=1$ for each $n$.

4. Theorem 3 of [6] has a natural extension for integral operators.

\section{REFERENCES}

[1] J.B. BAillon, Un Théorème de type ergodique pour les contractions non linéare dans un espace de Hilbert, C. R. Acad. Sci. Parıs Sér. A-B 280 (1975), A1511A1514.

[2] H. BRÉZIS A.ND F.E. BRowder, Remarks on Nonliner Ergodic Theory, Advances in Math. 25 (1977), 165-177.

[3] R.E. BRUCK, On the almost-convergence of iterates of a nonexpansive mapping in Hilbert space and the structure of the weak $\omega$-limit set, Israel J. Math. 29 (1978), 1-16.

[4] K. Goebel ANd W.A. Kirk, A fixed point theorem for asymptotically nonexpansive mappings, Proc. Amer. Math. Soc. 35 (1972), 171-174.

[5] G. H. HARDY, Divergent Series, Oxford Unıv. Press, 1949.

[6] N. HiRANo AND W. TAKAhashi, Nonliner Ergodic theorems for nonexpansive mappings in Hilbert spaces, Koda1 Math. J. 2 (1979), 11-25.

[7] Mattheiv Liu and B.E. Rhoades, Some properties of the Chebyshev method, Pacific J. Math. 80 (1979), 213-225.

[8] W.O. RAY, The fixed point property and unbounded sets in Hilbert space. Trans. Amer. Math. Soc. 258 (1980), 531-537.

[9] S. ReICH, Almost Convergence and Nonliner Ergodic Theorems, J. Approximation Theory 24 (1978), 269-272.

[10] B. E. RHOADES, Some applications of strong regularity to Markov Chains and fixed point theorems, to appear in "Approximation Theory, III", ed. E. W. Cheney, Academic Press (1980), 735-740.

INDIANA UNIVERSITY

BLOOMINGTON, INDIANA 47405

U.S.A. 